\title{
HospiT'Win: A Predictive Simulation-Based Digital Twin for Patients Pathways in Hospital
}

\author{
$1^{\text {st }}$ Abdallah Karakra \\ Industrial Engineering Department \\ University of Toulouse
}

IMT Mines Albi, Route de Teillet 81000 Albi, France

abdallah.karakra@mines-albi.fr

$3^{\text {rd }}$ Elyes Lamine

Industrial Engineering Department

University of Toulouse

IMT Mines Albi, Route de Teillet 81000 Albi, France

elyes.lamine@mines-albi.fr

\author{
$2^{\text {nd }}$ Franck Fontanili \\ Industrial Engineering Department \\ University of Toulouse \\ IMT Mines Albi, Route de Teillet 81000 Albi, France \\ franck.fontanili@mines-albi.fr
}

$4^{\text {th }}$ Jacques Lamothe

Industrial Engineering Department

University of Toulouse

IMT Mines Albi, Route de Teillet 81000 Albi, France

jacques.lamothe@mines-albi.fr

\begin{abstract}
Hospitals are starting to move away from traditional based-systems to the information technology based-systems. Today, Internet of Things (IoT), Body Sensor Network (BSN), Modeling, Simulation, and Artificial Intelligence (AI) are core technology elements that will be used in hospital of the future to improve the quality of patient care. Collecting the patient's data and monitoring their states and behavior became mandatory to improve their care. This paper proposes a novel framework for supporting the hospital of the future named HospiT'Win. This framework uses the core technology elements mentioned above to create a digital twin, that is a virtual replica of the hospital, allowing the health care providers to trace the patient's pathways data, monitor their behaviors, and predict their near future outcomes. So that, they can provide the right care in a right place, and in a right time. The paper explains in details the main components, the structure, and the way to synchronize the state and the behavior of the digital twin with the patients pathways in the real hospital. In case of unexpected events, HospiT'Win predicts the near future to see their impact on the real hospital. Moreover, it provides the possible solutions to minimize the impact of these events to preserve the quality of health care inside the hospital.

Index Terms-Digital Twin, Hospital of the Future, Internet of Things, Modeling, Simulation.
\end{abstract}

\section{INTRODUCTION}

Digital twin (DT) is attracting a lot of attention and hype in the recent years, and is being considered as a key research area. There are many scientific research papers that showed the importance of applying the digital twin in the modern industry [1]. At the best of our knowledge, no scientific research paper showed the importance of applying the digital twin in healthcare organizations for managing the patients pathways in hospitals. In scientific research, several definitions for the digital twin are existing, but there is no clear, specific and unique definition for this term [1] [2]. Most of the existing definitions relate to the life of a vehicle [3], future of manufacturing processes, and product life cycle management (PLM). This paper discusses in details its application in hospital, called HospiT'Win.
Digital twin is not exactly new. Actually, the core elements of a digital twin are already well known and exist such as modeling, simulation and internet of things (IoT). The idea behind the digital twin can be summarized as follows: there is (1) a real world, (2) a virtual world, (3) a link of data from the real world to the virtual world and (4) a link of information and knowledge from the virtual world to the real world as illustrated in Fig. 1.

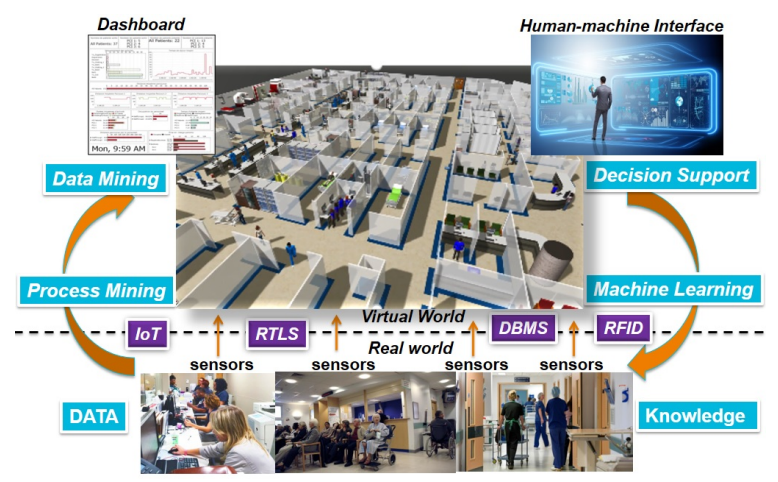

Fig. 1. Digital Twin

This paper aims to propose a new class of digital twin to support patient pathways in a hospital named HospiT'Win. In particular, the following questions are developed: What is a digital twin for patients pathways in hospital? What are the levels and the models that are used in the creation of HospiT'Win? How to link the HospiT'Win with the real hospital so that they can synchronize up-to-date data and information? How the HospiT'Win can predict the impact of unexpected events in near future? How can minimize the effect of this impact to preserve the quality of care from any future risks or problems?

This paper is organized as follows: literature review is provided in section II. Then the proposed approach is explained 
in section III and the conclusion in section IV.

\section{Literature REview}

The original idea behind the "twin" returned back to the NASA's Apollo project (National Aeronautics and Space Administrations), when NASA created two exact copies of space vehicles. The one that was on the earth was referred to as "twin", utilized to mirror the condition of the space vehicle during the mission [1] [4]. The concept of the Digital Twin has been used in Product Lifecycle Management (PLM), in a previous work at the University of Michigan in 2002 in a presentation for Grieves [5]. Today, in scientific literature, there are many explanations and definitions for the digital twin. Those definitions are related to the life of a vehicle, future of manufacture, and product lifecycle management (PLM).

First definitions of digital twin came for a vehicle and have been extended to products: B.T. Gockel et al. [6] define the digital twin as "Ultra-realistic, cradle-to-grave computer model of an aircraft structure that is used to assess the aircrafts ability to meet mission requirements". For K. Reifsnider et al. [7], the digital twin is "Ultra-high fidelity physical models of the materials and structures that control the life of a vehicle". For Y. Bazilevs et al [8], the digital twin is "High- fidelity structural model that incorporates fatigue damage and presents a fairly complete digital counterpart of the actual structural system of interest". Other definitions and explanations for the digital twin are in [9], [10], [11], [12].

The most common definition used today for the digital twin of a product is given by Glaessgen, Stargel (2012) "digital twin is an integrated multi-physics, multi-scale, probabilistic simulation of a complex product and uses the best available physical models, sensor updates, etc., to mirror the life of its corresponding twin." [13].

In short, there are many definitions in the scientific literature for the digital twin. Most of these definitions agree that the digital twin should have the following core elements: Model, Simulation, Sensors, and IoT. This paper exploits those core elements to describe our proposed approach.

\section{Proposed Approach}

This section is divided into two parts. The first part illustrates in details the framework of HospiT'Win. The second part proposes a way to link and to synchronize the HospiT'Win with real hospital in the near-real time.

\section{A. HospiT'Win: a digital twin for patients pathways in hos- pital}

HospiT'Win is a new class of digital twin. It proposes to support the patient pathways in hospital of the future. It is composed of three levels of functionalities: construction and validation level, tracking and monitoring level, and vision of the future level as illustrated in Fig. 2.

The three levels presented in Fig. 2 are differing in the way of data integration between the real sensor objects and the digital model. At the validation level, a meta-model is configured manually and not connected or synchronized with

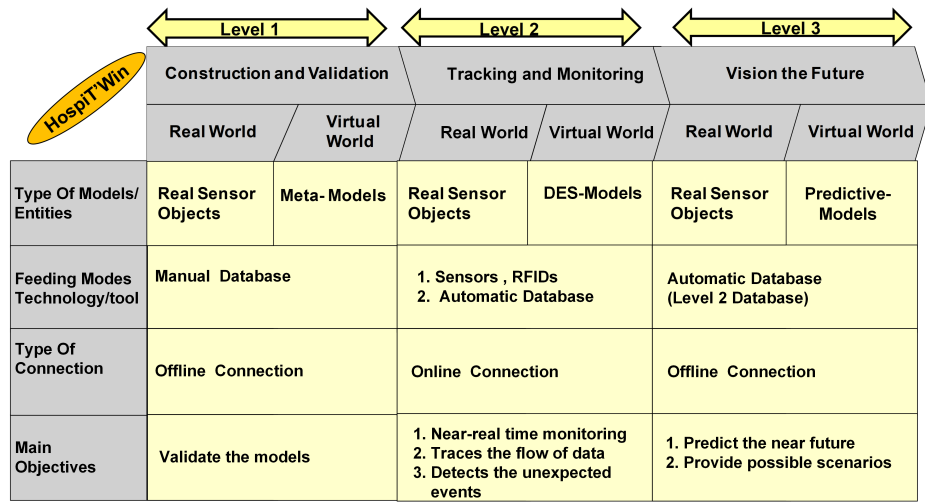

Fig. 2. HospiT'Win Levels

any data or real sensor objects directly. This type of connection can be called "offline" connection. The model is manually fed by data extracted from hospital databases. It enables to specify the perimeter of the digital twin, list of concerned resources, granularity and precision of the models, associate sensors to events. In level two, the discrete event simulation model (DESmodel) is connected and synchronized to work in the near-real time with real sensor objects. This connection can be called "online" connection. Data are captured by the sensors to feed a synchronized model. The predictive model in the third level is not synchronized to the real world. It is used to simulate the near future in case of unexpected events from the current state of the real world. This state is based on the flow of data received from the database that exists at the second level.

For more clarification, this paper would like to discuss each level separately. It will discuss in details the models, the type of connections and the techniques utilized in each level.

Construction and Validation level: At this level, there is no actual connection or synchronization between the virtual world (virtual hospital) and the real world (real hospital). In other words, any change in the state of the real hospital have no effect on the virtual hospital and vice versa. Fig. 3 shows the main parts of this level.

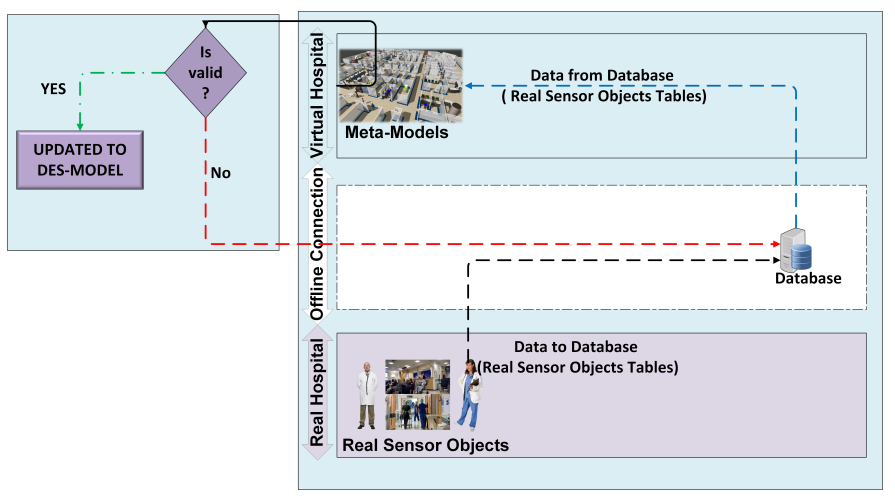

Fig. 3. Construction and validation level

This level is composed of real sensor objects in the real world, digital meta-models in the virtual world, and the 
database contains data and information about each real sensor object in the real world.

Real sensor objects is any entity inside the real hospital that has a sensing capability, so that the sensors can capture/collect near-real time data about this entity.

In this paper, the digital meta-model is defined as a virtual representation of the real sensor objects with a set of concepts. It is typically used to describe what a real sensor object will look like, and what are the states and behaviors of this object. It just describes the parts and the components of the real sensor object and how they interact with each other. There are no automated data or information exchange between the meta-model and the real sensor object. The database is the only technique used to feed the meta-model with data and information about the real sensor object.

The main function for this level is to construct a metamodel for the real sensor objects as initial developments and to validate if the models are correctly implemented to have the same state and behavior as the real sensor objects. After that, the meta-model is used to build the Discrete Event Simulation living models in the second level.

Tracking and monitoring level: Fig. 4 illustrates a detail picture of the second level (tracking and monitoring level). This online level is composed of real sensor objects, IoT devices (including wireless sensor networks (WSNs), radiofrequency identification (RFID)) and multi-processing layers (including preparing and cleaning data layer, security layer, and dynamic database) to synchronize the virtual world (DESmodel) to the real world. The DES-model is a digital living

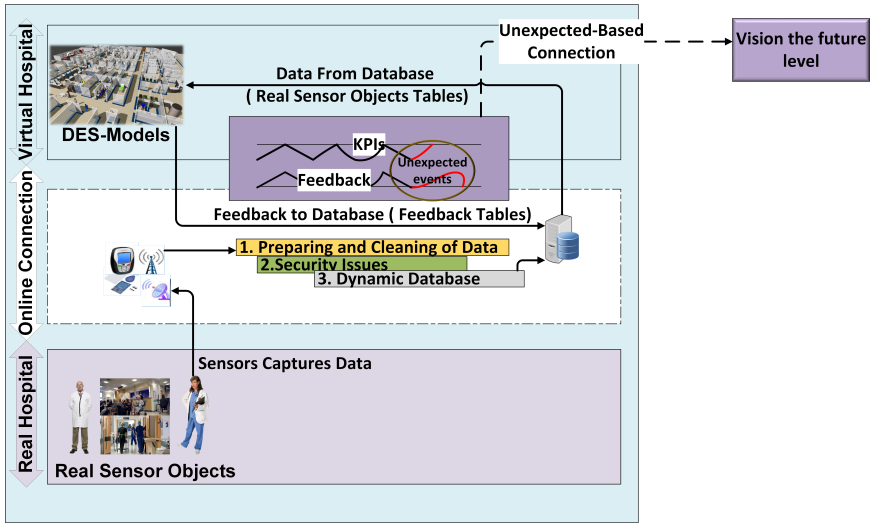

Fig. 4. Tracking and Monitoring level

model, in which the data flow in one way from the real sensor object to the digital object based on a certain event. This model reflects the behavior and the state of the real sensor object in the near-real time. Any updates and changes on the state and behavior of the real sensor object leads to update the state and behavior of the digital object in the near-real time. This level contains three processing layers to prepare the data before transferring from the real sensor objects to the digital models as follows:

1) Data preparing and cleaning layer: the responsibility of this layer is to clean and to prepare the data before sending to the DES-model. Actually, the data provided by the sensors are potentially unreliable and need to be cleaned before their use [14], [15].

2) Security layer: the responsibility of this layer is to ensure that the data transfer from the real sensor objects to the DES-models are secure [16].

3) Dynamic Database layer: to store the updated data and information. DES-model uses the database to monitor and to track the flow of data from the real sensor objects to the digital models. It stores the feedback about the behavior of the models to confirm that it correctly works with respect to the real hospitals KPIs (Key Performance Indicators) which make easier to detect the risk and fix before occurring.

The main purpose for this level are summarized as follows:

- Uses for near-real time monitoring (like a live video): it provides information about the near-real time state and behavior of the real sensor objects (flow of patients, operating room monitoring, registration desk, etc.).

- Tracks the flow of data from the real world to the virtual world.

- Detects the unexpected situation. For example, before the problem occurs it generates an event to call the predictive model to see the future (delay, change in schedule,.. etc).

Vision of the future level: This level is composed of predictive model, stochastic simulation, and artificial intelligence (AI) techniques as demonstrated in Fig. 5. In this

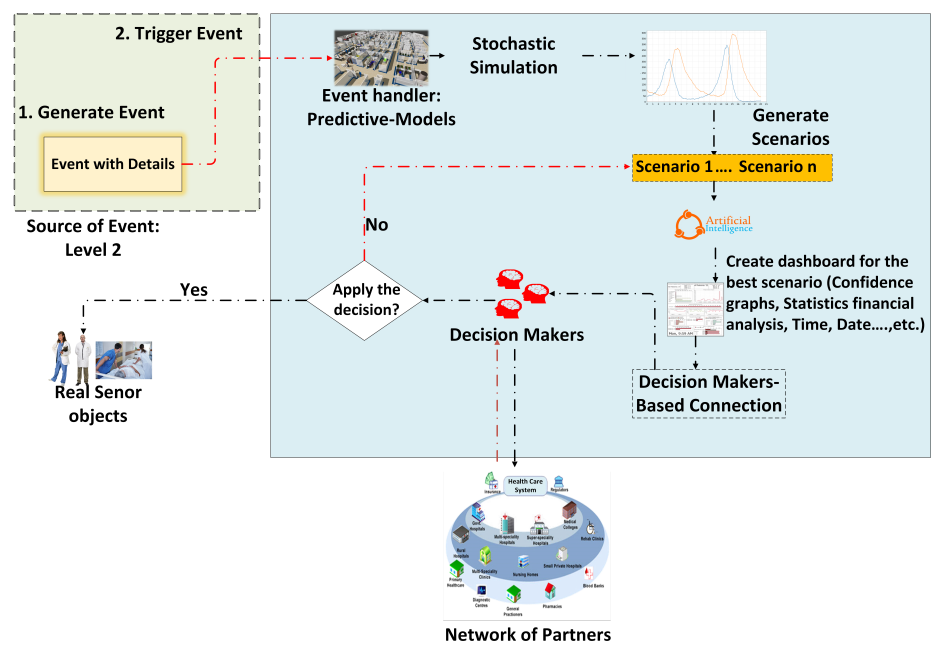

Fig. 5. Vision of the Future Level

paper, predictive model is a digital representation that applies stochastic simulation techniques on the near real time data and information that receives from the level two database. In case of unexpected events, it predicts the near future outcomes to see the impact of these events in the real hospital. Also, it generates various future scenarios to minimize the impact of these events. It uses artificial intelligence techniques to determine the acceptable scenario to adopt in the real hospital with many details about the risks, financial, etc. The following steps shows the main functions for this level. 
1) In case of unexpected issues in level two, unexpected event is generated.

2) Level two triggers the generated event with details about the issues.

3) The predictive digital model handles the event by applying the stochastic simulation on the event's data to predict the near future with possibility to generate scenarios to minimize or to solve the impact of these issues.

4) AI techniques use to select the best applicable scenario to minimize the impact of these issues in the real hospital.

5) Before applying the selected scenario in the real hospital, connection is opened with the decision makers. Decision makers take their decision based on the available knowledge and performance of the selected scenario.

6) Decision makers can contact external partners (pharmacies, ambulances, other hospitals, etc.) to be confident with a decision that is proposed.

7) Based on the confident percentage the decision could apply or not.

\section{B. Linkage between the virtual hospital and the real hospital}

The paper proposes to use a database to synchronize the virtual hospital with the real hospital in a near-real time and based on discrete events (DE).

The following steps summarize the way to link and synchronize the virtual hospital with the real one.

1) Create a database that contains the following types of tables:

- Tables to store data, information, state, behavior, etc. about each real sensor object (patients, doctors, etc.).

- Tables to store feedback that come from the DESmodels.

- Tables for hospital KPIs (length of stay, staff satisfaction, patients satisfaction, etc.).

2) Thanks to the WSNs, and RFIDs, data are collected about the hospital real sensor objects and send to the database over the internet.

3) Updated data from the database are pushed to and read by the virtual hospital (open connection to the database).

4) The feedback data transfer from the virtual hospital to the database (feedback tables updated).

Those technologies could be used to make the connection: MySQL database, ODBC driver, and Windows Data Source.

\section{CONCLUSION}

This paper proposed a novel framework to support the hospital of the future named HospiT'Win. In case of unexpected events in the real hospital, this framework can predict the near future to see what will be the impact on the hospital and what are the possible solutions to reduce this impact. Moreover, the paper demonstrated a way to connect the HospiT'Win with real hospital; This allows to track, monitor and validate that the hospital functionality goes in the right direction and in a right time.

\section{REFERENCES}

[1] E. Negri, L. Fumagalli, and M. Macchi, "A review of the roles of digital twin in cps-based production systems," Procedia Manufacturing, vol. 11, pp. 939-948, 2017.

[2] W. Kritzinger, M. Karner, G. Traar, J. Henjes, and W. Sihn, "Digital twin in manufacturing: A categorical literature review and classification," IFAC-PapersOnLine, vol. 51, no. 11, pp. 1016-1022, 2018.

[3] M. Shafto, M. Conroy, R. Doyle, E. Glaessgen, C. Kemp, J. LeMoigne, and L. Wang, "Modeling, simulation, information technology \& processing roadmap," National Aeronautics and Space Administration, 2012.

[4] B. A. Talkhestani, N. Jazdi, W. Schlögl, and M. Weyrich, "A concept in synchronization of virtual production system with real factory based on anchor-point method," in 11th CIRP Conference on Intelligent Computation in Manufacturing Engineering, 2017.

[5] A. Karakra, F. Fontanili, E. Lamine, J. Lamothe, and A. Taweel, "Pervasive computing integrated discrete event simulation for a hospital digital twin," in 2018 IEEE/ACS 15th International Conference on Computer Systems and Applications (AICCSA). IEEE, 2018, pp. 1-

[6] B. Gockel, A. Tudor, M. Brandyberry, R. Penmetsa, and E. Tuegel, "Challenges with structural life forecasting using realistic mission profiles," in 53rd AIAA/ASME/ASCE/AHS/ASC Structures, Structural Dynamics and Materials Conference 20th AIAA/ASME/AHS Adaptive Structures Conference 14th AIAA, 2012, p. 1813.

[7] K. Reifsnider and P. Majumdar, "Multiphysics stimulated simulation digital twin methods for fleet management," in 54th AIAA/ASME/ASCE/AHS/ASC Structures, Structural Dynamics, and Materials Conference, 2013, p. 1578.

[8] Y. Bazilevs, X. Deng, A. Korobenko, F. L. di Scalea, M. Todd, and S. Taylor, "Isogeometric fatigue damage prediction in large-scale composite structures driven by dynamic sensor data," Journal of Applied Mechanics, vol. 82, no. 9, p. 091008, 2015.

[9] T. Gabor, L. Belzner, M. Kiermeier, M. T. Beck, and A. Neitz, "A simulation-based architecture for smart cyber-physical systems," in $\mathrm{Au}$ tonomic Computing (ICAC), 2016 IEEE International Conference on. IEEE, 2016, pp. 374-379.

[10] E. M. Kraft, "The air force digital thread/digital twin-life cycle integration and use of computational and experimental knowledge," in 54th AIAA Aerospace Sciences Meeting, 2016, p. 0897.

[11] A. Canedo, "Industrial iot lifecycle via digital twins," in Proceedings of the Eleventh IEEE/ACM/IFIP International Conference on Hardware/Software Codesign and System Synthesis. ACM, 2016, p. 29.

[12] M. Schluse and J. Rossmann, "From simulation to experimentable digital twins: simulation-based development and operation of complex technical systems," in Systems Engineering (ISSE), 2016 IEEE International Symposium on. IEEE, 2016, pp. 1-6.

[13] F. Tao, J. Cheng, Q. Qi, M. Zhang, H. Zhang, and F. Sui, "Digital twindriven product design, manufacturing and service with big data," The International Journal of Advanced Manufacturing Technology, vol. 94, no. 9-12, pp. 3563-3576, 2018.

[14] S. R. Jeffery, G. Alonso, M. J. Franklin, W. Hong, and J. Widom, "Declarative support for sensor data cleaning," in International Conference on Pervasive Computing. Springer, 2006, pp. 83-100.

[15] Y. Zhuang, L. Chen, X. S. Wang, and J. Lian, "A weighted moving average-based approach for cleaning sensor data," in Distributed Computing Systems, 2007. ICDCS'07. 27th International Conference on. IEEE, 2007, pp. 38-38.

[16] Q. Wang, K. Ren, S. Yu, and W. Lou, "Dependable and secure sensor data storage with dynamic integrity assurance," ACM Transactions on Sensor Networks (TOSN), vol. 8, no. 1, p. 9, 2011. 